\title{
Ciclo de mejora en el aula aplicado en una asignatura clínica con pacientes del Postgrado en Odontología
}

\section{Improvement cycle in classroom applied to a clinic subject with patients of the Dentistry Postgraduate Degree}

Jenifer MARTín GonzÁLEZ

ORCID: https://orcid.org/0000-0001-9282-133X

Universidad de Sevilla

Departamento de Estomatología

jmartin30@us.es

DOI: http://dx.doi.org/10.12795/9788447231003.045

Pp.: 947-963 


\section{Breve descripción del contexto}

La asignatura Clínica en Operatoria Dental y Endodoncia perteneciente al Máster Oficial en Odontología Restauradora, Estética y Funcional se imparte de forma anual, siendo coordinadora de la misma en el presente curso académico. Tiene una carga lectiva de 12 créditos prácticos, realizándose en la totalidad de los créditos prácticas clínicas (actividad asistencial a pacientes). La asignatura se imparte los lunes de $16 \mathrm{~h}$ a $20 \mathrm{~h}$ en dos grupos de prácticas al mismo tiempo con un total de 20 estudiantes, en el área prácticas clínicas de la Facultad de Odontología, la cual está organizada por gabinetes dentales o boxes clínicos en los que el alumnado trabaja en parejas.

\section{Diseño previo del CIMA}

\section{Mapa de contenidos y problemas claves que describa los aprendizajes deseables y los núcleos de trabajo en el aula}

El mapa de contenidos diseñado se muestra a continuación:

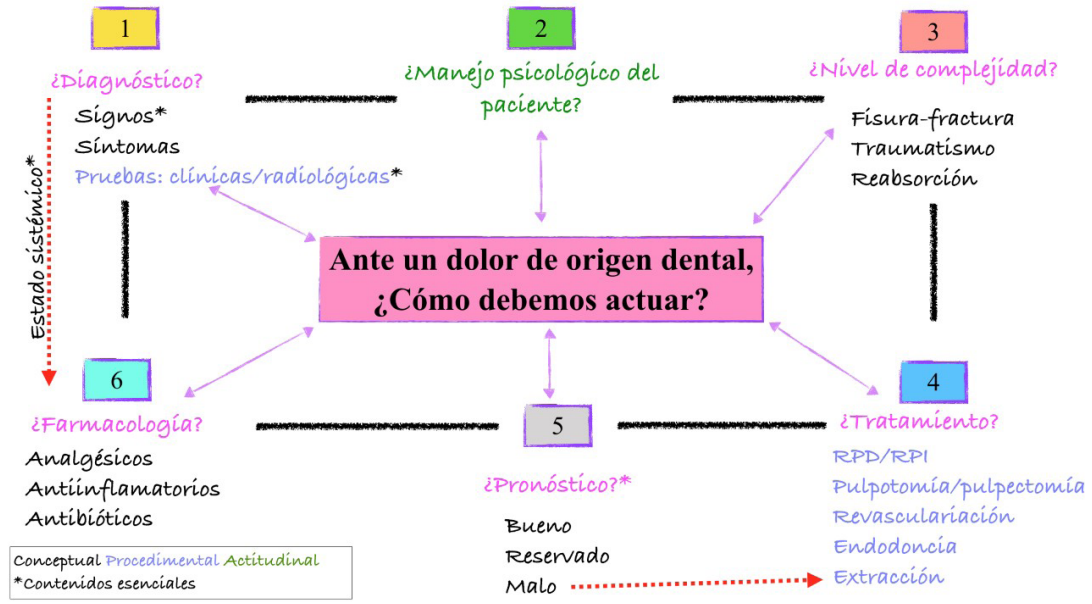

Figura 1. Contenidos de las prácticas clínicas Ciclos de Mejora en el Aula (2020). Experiencias de Innovación Docente de la US 
La asignatura no tiene bloques temáticos en sí; la temática a abordar depende de la patología que presente cada paciente. El dolor dental es uno de los motivos más frecuente por el que los y las pacientes demandan asistencia en esta asignatura, de ahí que constituya el contenido estructurante $u$ organizador en el centro del mapa.

Este contenido estructurante vincula el conocimiento de los aspectos esenciales para realizar el diagnóstico de la enfermedad (número 1 del mapa), manejar psicológicamente al paciente (número 2 del mapa), identificar el nivel de complejidad del caso (número 3 del mapa), planificar el tratamiento (número 4 del mapa), determinar el pronóstico (número 5 del mapa) y establecer pauta farmacológica en caso de que fuera necesaria (número 6 del mapa).

A su vez en cada uno de los apartados numerados se recogen los contenidos estructurantes de los mismos.

Por último, se han diferenciado en colores los contenidos conceptuales de los procedimentales y actitudinales.

\section{Modelo metodológico posible y secuencias de actividades programadas, partiendo de los problemas seleccionados.}

El modelo metodológico posible se aplicó en dos sesiones. Cada sesión tiene una duración de 4 horas. Se llevaron a cabo 3 fases que se muestran a continuación con la secuencia de actividades programadas en cada una de las fases:

Ciclos de Mejora en el Aula (2020). Experiencias de Innovación Docente de la US Esta obra se distribuye con la licencia Creative Commons 
- Fase 1a: 1 hora y 40 minutos, divididas en dos sesiones de 45 minutos cada día. Esta fase consiste en un seminario preclínico donde el aprendizaje está basado en supuestos casos clínicos que abordarán los contenidos del mapa conceptual y que se han reflejado en el cuestionario inicial que el alumno realiza.

-Fase 2: 6 horas, divididas en dos sesiones de 3 horas cada día. Desarrollo de las prácticas clínicas con atención a pacientes reales.

-Fase 3â: 20 minutos, divididos en dos sesiones de 10 minutos cada día. Puesta en común del desarrollo de las prácticas clínicas (aplicación de lo planteado en la fase 1, complicaciones y su abordaje, etc).

Figura 2. Fases del modelo metodológico posible

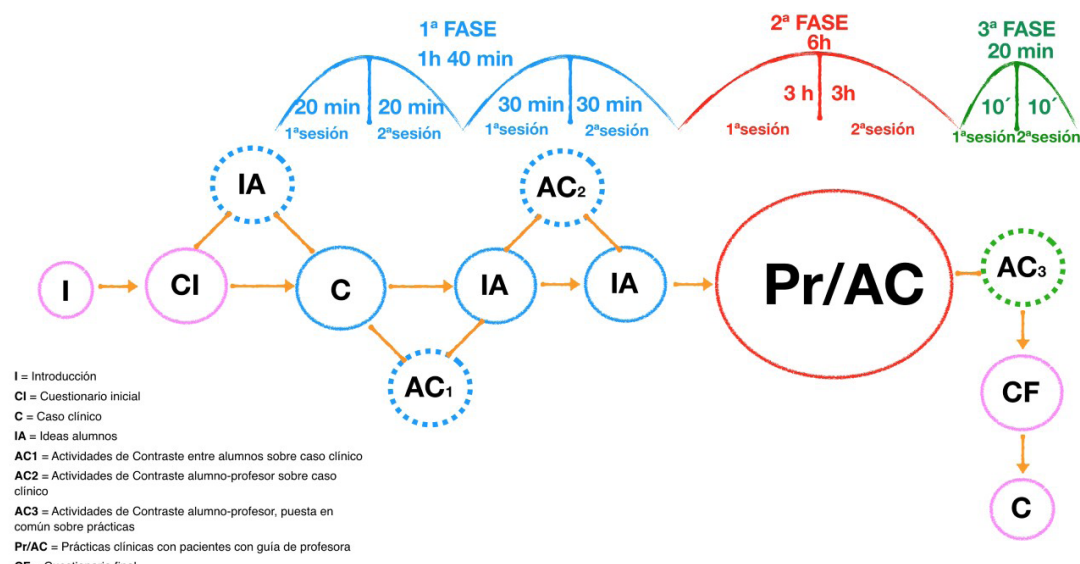

$c=$ conclusiones

Figura 3. Modelo metodológico posible

\section{$1^{\mathrm{a}}$ FASE}

I ACTIVIDAD 1: Introducción y cuestionario inicial

Información del objetivo del cuestionario inicial, resaltando la importancia que no es un examen y es totalmente anónimo

En el cuestionario se les plantea a través de la formulación de las preguntas todos los aspectos,

Cl contenidos y problemas que se reflejan en el mapa de contenidos y que se van a abordar en las diferentes fases que se plantean en el modelo metodológico.

ACTIVIDAD 2: Respuesta individual 5 minutos (en cada sesión)

IA Reflexión de los alumnos sobre las respuestas que dieron (y recuerdan) en el cuestionario a nivel "individual e intercambian opiniones entre compañeros.

ACTIVIDAD 3: Caso clínico 15 minutos (en cada sesión)

Asignación, lectura y análisis de casos clínicos. A través de casos clínicos se crean problemas centrados y directos que simularán casos reales, generando enigma y suscitando el interés de los

alumnos. Los alumnos trabajarán en grupos por parejas que ya tienen asignadas para el resto de

C la actividad del máster, habiendo un total de 10 grupos. Se asignarán 2 casos clínicos por escrito (1 por sesión) idénticos a cada pareja, en los que se realizarán una serie de preguntas sobre los mismos siguiendo los aspectos planteados en el mapa conceptual sobre diagnóstico, manejo psicológico del paciente, complejidad del caso, tratamiento a realizar, pronóstico y farmacologia si fuese necesaria. Pueden consultar los recursos que necesiten (bases científicas, apuntes de asignaturas, etc) así como formularme cualquier duda que les puedan surgir.

Figura 4. Actividades programadas en fase 1a

Ciclos de Mejora en el Aula (2020). Experiencias de Innovación Docente de la US Esta obra se distribuye con la licencia Creative Commons Reconocimiento-NoComercial-SinObraDerivada Internacional (CC BY-NC-ND 4.0.) 
Se llevará a cabo la atención a los pacientes. Se intentará seleccionar pacientes cuyo tratamiento sea el abordado en el mapa de contenidos aunque inevitablemente depende de la demanda asistencial de los pacientes que acudan los días en los que se va a aplicar el CIMA. A pesar de ello, al haber seleccionado una situación bastante frecuente en el perfil de pacientes que se tratan en la asignatura, de forma previsible muchos alumnos podrán tener un paciente con el aplicar los contenidos abordados en la fase 1a.

En esta actividad se realiza la atención a los pacientes por parejas. Los alumnos deben de realizar los procedimientos necesarios y aplicar en los pacientes los aspectos abordados durante la fase 1․

Cada paso en el abordaje del paciente tiene que estar revisado y supervisado por mí por lo que los alumnos deben consultarme cualquier duda que pueda surgirles y cualquier decisión antes de ejecutar el tratamiento, por lo que la interacción alumno-profesora es continua durante la totalidad de las prácticas.

Figura 5. Actividades programadas en fase 2a

$3^{\mathrm{a}}$ FASE

CF

Información del objetivo del cuestionario final, resaltando la importancia que no es un examen y es totalmente anónimo.

\title{
Cuestionario inicial-final para hacer un seguimiento de la evolución de los estudiantes en relación con los problemas y contenidos claves.
}

\author{
Las preguntas "problemas" relacionadas con el mapa \\ de contenidos fueron las siguientes:
}

Ciclos de Mejora en el Aula (2020). Experiencias de Innovación Docente de la US Esta obra se distribuye con la licencia Creative Commons Reconocimiento-NoComercial-SinObraDerivada 


\section{Ante un dolor dental, ¿Cómo debemos actuar?}

Este ejercicio que estás leyendo no es un examen. Estamos realizando un estudio sobre el aprendizaje de los alumnos en el Máster Oficial que estás cursando. Nos interesa mucho saber cómo actuarías ante la supuesta situación clínica que se plantea.

Responde a las siguientes preguntas sin consultar absolutamente nada. Recuerda: ¡Esto no es un examen! y, para tu tranquilidad, responde de forma anónima, utilizando un nombre inventado o pseudónimo.

Pseudónimo:

Imagínate que estás un día cualquiera en tu clínica y acude de urgencias un paciente que te dice que le duele mucho una muela y que no ha podido dormir en toda la noche.

1. ¿Cómo puedes saber de qué diente procede el dolor? Explica con detalle tu actitud y actuación en este caso.

2. ¿Qué tipo de dolor presenta el paciente?

Problema 2 (Manejo psicológico del paciente)

3. El paciente está muy nervioso y se muestra muy ansioso ante lo que le está ocurriendo, ¿Crees que esto influye en lo patología que presenta y en el abordaje del posible tratamiento?

Problema 3 (Casos complejos)

4. Si llegado el caso decides radiografiar la pieza afectada y observas una imagen radiolúcida en el tercio medio radicular de la raíz palatina compatible con reabsorción interna, ¿Harías alguna prueba complementaria adicional? ¿Qué implicación crees que tiene esto en el diagnóstico y plan de tratamiento?

Problema 4 (Tratamiento)

5. Teniendo en cuenta el caso anterior ¿Qué tratamiento propondrías? Explica en qué consistiría

Problema 5 (Pronóstico)

6. Tras tu diagnóstico y posible tratamiento, lógicamente el paciente antes de decidir si se va a tratar, quiere saber cuánto le va a durar el diente en boca, ¿Qué le dirías? ¿Cómo le asesorarías?

Problema 6 (Farmacologia)

7. Vaya!!!!, un imprevisto... el paciente es alérgico a la penicilina!!!!, te va a preguntar qué debe tomar para el dolor, ¿Qué pauta farmacológica le prescribirías? Duración, posología, dosis, etc.

Figura 7. Cuestionario inicial-final

El cuestionario tanto inicial como final se entregaron y se completaron de forma presencial previo y al final de la aplicación del CIMA respectivamente. La totalidad de los alumnos matriculados (20) en esta asignatura lo realizaron.

Ciclos de Mejora en el Aula (2020). Experiencias de Innovación Docente de la US Esta obra se distribuye con la licencia Creative Commons 


\section{Aplicación del CIMA}

\section{Relato de las sesiones}

\section{A) Descripción y análisis de las sesiones.}

Tal como estaba diseñado el CIMA, se desarrolló en 2 sesiones idénticas de 4 horas cada una. En el comienzo de la primera sesión se explica de forma detallada al alumnado todo el proceso que se va a realizar. Previo a esta sesión, el alumnado realiza el cuestionario inicial (Fase 1, Actividad 1) donde se le plantea a través de la formulación de las preguntas todos los aspectos, contenidos y problemas que se reflejan en el mapa de contenidos y que se van a abordar en las dos fases que se plantean en el modelo metodológico del CIMA. Así al inicio de la primera fase o sesión, durante los primeros 15 minutos los alumnos y alumnas reflexionan sobre las respuestas que dieron (y recordaban) en el cuestionario a nivel individual e intercambian opiniones entre compañeros y compañeras (Fase 1 , Actividad 2). Esta parte se alargó más de lo planificado (5 minutos), ya que había respuestas diferentes entre ellos y ellas y, estaban muy animados y animadas a debatir, lo que resultó muy interesante.

Posteriormente, se procedió a la asignación, lectura y análisis del caso clínico (Fase 1, Actividad 3). Los alumnos y alumnas se pusieron a trabajar por parejas y a redactar las respuestas en cada plantilla entregada a nivel individual (Fase 1, Actividad 4). Llama la atención la interacción tan positiva que tienen entre ellos y ellas y la gran cantidad de dudas que les van surgiendo en aspectos claves de lo preguntado en el caso clínico. Durante este tiempo me voy acercando a cada pareja de trabajo y me van consultando las cuestiones que se les genera y las voy diciendo

Ciclos de Mejora en el Aula (2020). Experiencias de Innovación Docente de la US Esta obra se distribuye con la licencia Creative Commons 
en alto generando nuevas cuestiones y poniendo ejemplos similares para que les llegue al resto de parejas de trabajo como actividad de contraste.

A continuación, voy nombrando cada pregunta y un o una portavoz de cada pareja comparte lo que han concluido sobre la misma (Fase 1, Actividad 5). Se generan dudas y debate en grupo prácticamente en cada pregunta. Ellos y ellas ponen de manifiesto no solo los conocimientos que van adquiriendo, sino la maduración de una visión global e integrada del paciente conectando la clase teórica que habían recibido por otro profesor de la asignatura la semana anterior sobre diagnóstico con el caso clínico planteado. Tras ello, les expongo la resolución del caso clínico planteado conectándolo con las respuestas que ellos y ellas habían dado previamente y con la evidencia científica actual al respecto (Fase 1, Actividad 6). Ellos y ellas se muestran muy atentos y atentas en toda esta parte y van anotando en su plantilla las respuestas que difieren con las contestadas por ellos y ellas anteriormente (Fase 1, Actividad 7). El primer día, se ocasionó un retraso de casi media hora que supuso un pequeño caos en la siguiente actividad ya que los y las pacientes estaban citados y citadas a las 16h y no pasaron a gabinete hasta casi las 16:30h.

La práctica clínica con pacientes reales ocupa la mayor parte del tiempo programado para el CIMA (3 horas). En esta segunda fase, los alumnos y alumnas intentan integrar los conocimientos adquiridos y aplicarlos a los pacientes que se les han asignado. Pesa a intentar, seleccionar pacientes con casos similares al que se había trabajado en las actividades anteriores para que los alumnos y alumnas pudieran aplicar todo lo aprendido en las actividades anteriores, 3 de las 10 parejas de trabajo no les llegó paciente con caso real similar al analizado. Durante

Ciclos de Mejora en el Aula (2020). Experiencias de Innovación Docente de la US Esta obra se distribuye con la licencia Creative Commons 
todas las prácticas, los alumnos y alumnas realizaron los procedimientos necesarios y aplicaron en los pacientes los aspectos abordados durante la fase anterior. Surgieron dudas en diferentes pasos del abordaje del paciente que se fueron resolviendo sobre la marcha conectándolo con la sesión previa. Los alumnos y alumnas se mostraban muy motivados y motivadas al ratificarse tanto en conocimientos conceptuales como en procedimientos psicomotrices. Estas prácticas deben terminar a las 19h, pero en ninguno de los dos días en los que se llevó a cabo el CIMA, se terminó a la hora prevista, lo que supuso retraso en la última actividad.

Al finalizar las prácticas clínicas, se llevó a cabo la puesta en común en grupo del desarrollo de las mismas (Fase 3, Actividad 1). Para ello, cada pareja expuso a sus compañeros y compañeras lo realizado en las prácticas con su paciente reflexionando sobre las fortalezas y debilidades que se habían podido encontrar en el transcurso de las prácticas. Yo actué como moderadora dando paso a cada pareja para exponer y resaltando los aspectos principales más importantes de lo expuesto por las diferentes parejas a modo conclusiones. Se superó el tiempo previsto para esta actividad (10 minutos) los dos días de aplicación del CIMA, estando más de media hora en esta etapa. Al finalizar las conclusiones, algunos y algunas de ellos o ellas me piden si puedo mandarles un borrador con las conclusiones de cada día, a lo que accedo y les mando por correo-e.

\section{B) Valoración: fortalezas y debilidades del CIMA.}

El alumnado a pesar del cansancio de las 4 horas seguidas de cada sesión, se muestra muy contento y motivado con la nueva dinámica. En general, las sensaciones 
son muy buenas y positivas. Supone para ellos y para ellas un gran reto y mayor esfuerzo que de costumbre o con la dinámica convencional donde no tienen que pensar antes de las prácticas y llegan directamente a atender a los pacientes. Asimismo, el alumnado se muestra muy receptivo ya que ve de forma inmediata como gana confianza en sí mismo para abordar al paciente de forma integral. Como debilidad destaco la organización del CIMA en cuanto a tiempo asignado a cada fase del mismo ya que en todas y en los dos días de aplicación hubo retraso generando esto estrés en el alumnado que se mostraba inquieto al saber que ya los pacientes les estaban esperando o al tenerse que quedar más tarde de lo habitual para la puesta en común tras las prácticas.

\section{Evaluación del aprendizaje de los estudiantes, comparando sus conocimientos iniciales y finales $y$, en su caso, presentando las Escaleras de Aprendizaje y el Cuadro de Evolución de cada alumno.}

Se compararon los cuestionarios iniciales y finales para establecer la evolución de las escaleras de aprendizaje. A continuación, se muestran escaleras de aprendizaje extraídas de dos bloques conceptuales trabajados durante la aplicación del CIMA:

Ciclos de Mejora en el Aula (2020). Experiencias de Innovación Docente de la US Esta obra se distribuye con la licencia Creative Commons 
PREGUNTA 7. ¡Vaya! ¡Un imprevisto! El paciente es alérgico a la penicilina. Te va a preguntar qué debe tomar para el dolor, ¿Qué pauta farmacológica le prescribirías? Duración, posología, dosis, etc.

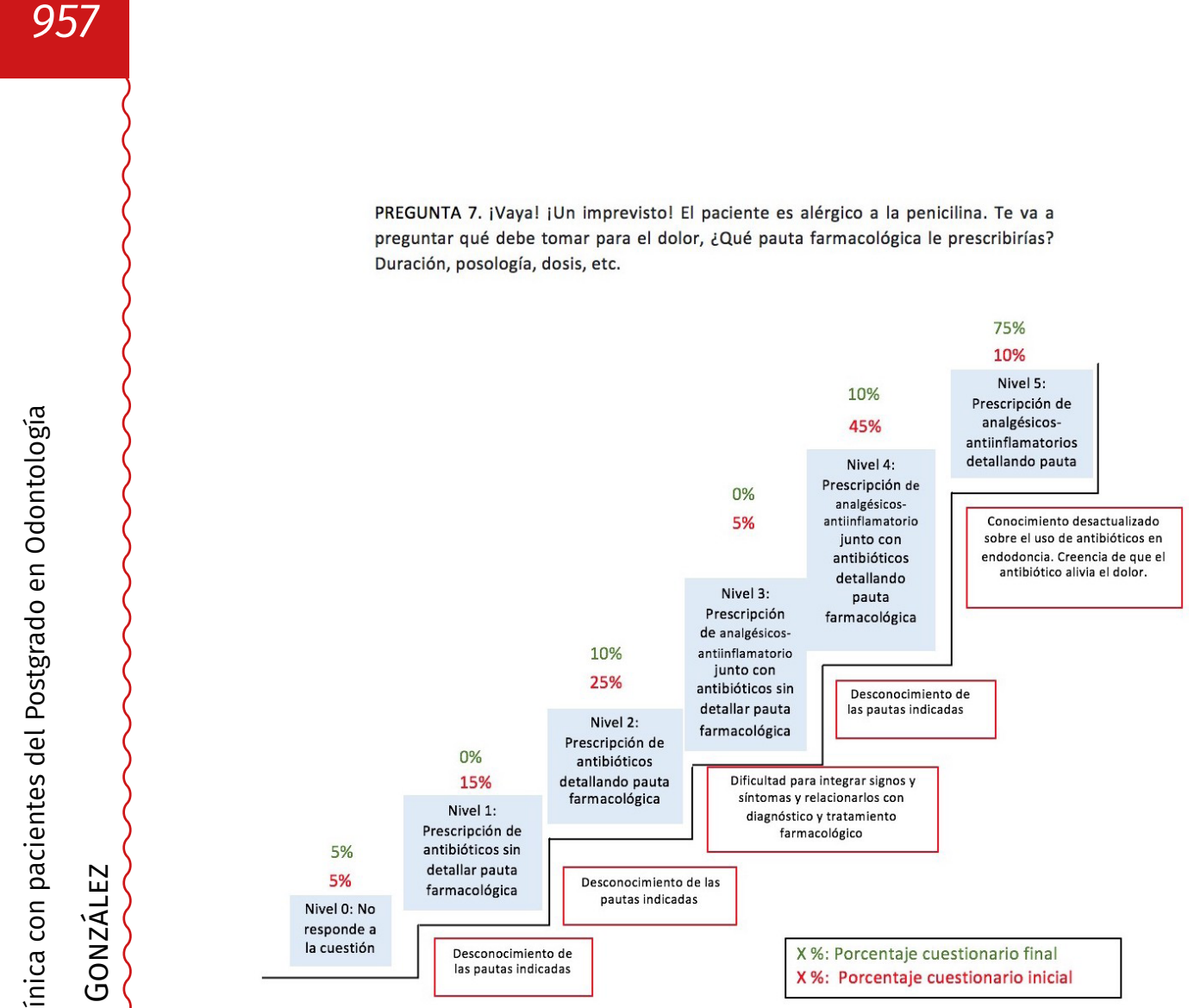

Figura 8. Escalera de aprendizaje 1

Ciclos de Mejora en el Aula (2020). Experiencias de Innovación Docente de la US (c) () $\odot$ Esta obra se distribuye con la licencia Creative Commons 
PREGUNTA 1. ¿Cómo puedes saber de qué diente procede el dolor? Explica con detalle tu actitud en este caso.

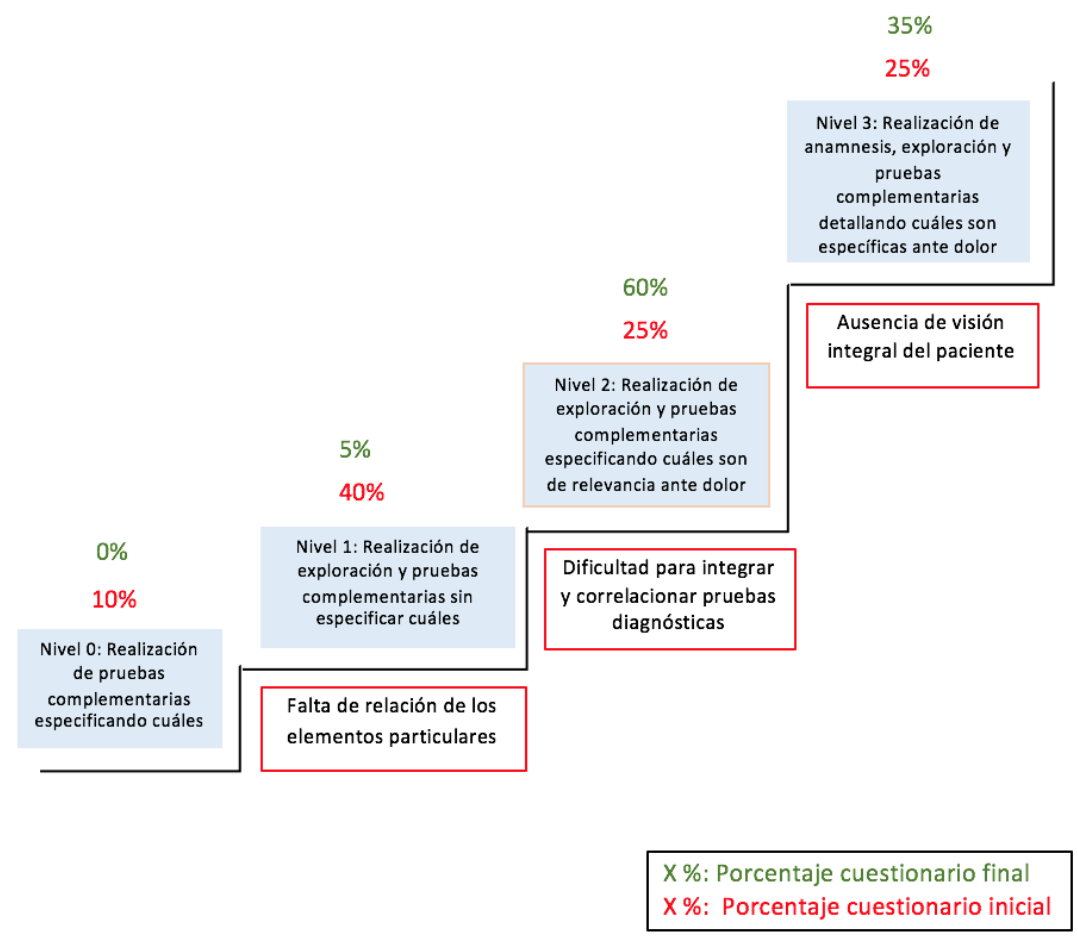

Figura 9. Escalera de aprendizaje 2

Como puede apreciarse, el impacto del modelo metodológico ha sido muy significativo. Lo más llamativo ha sido que los alumnos han sido capaces de interrelacionar todos los contenidos abordados mediante este modelo metodológico.

Ciclos de Mejora en el Aula (2020). Experiencias de Innovación Docente de la US Esta obra se distribuye con la licencia Creative Commons 


\section{Evaluación del CIMA}

\section{Cuestiones a mantener y cambios a introducir para un futuro Ciclo de Mejora más amplio}

Para un futuro CIMA, las cuestiones a mantener son todas las referentes al desarrollo de las sesiones de trabajo, es decir, la estructura de las prácticas y la incorporación de las sesiones pre y post a las mismas con puesta común en grupo. Teniendo en cuenta lo expuesto anteriormente como debilidad, lo llevaría a cabo con un número mayor de horas para que se trabajara todo lo planteado y llevado a cabo en este CIMA, pero de forma más extendida y sin prisas por los retrasos observados en éste. Me he dado cuenta que conforme el alumnado construye su propio conocimiento de forma madurada y gana confianza, le surgen muchas más inquietudes y ganas de seguir avanzando y eso requiere tiempo. Por otro lado, teniendo en cuenta que, a pesar de tener los conocimientos suficientes y necesarios para llevar a cabo ciertos procedimientos psicomotrices, muchos alumnos y alumnas se enfrentan por primera vez a los mismos directamente en pacientes lo que les inquieta mucho por lo que plantearía algún tipo de taller pre-clínico, sobre dientes naturales sobre los que poder simular y realizar estos procedimientos psicomotrices antes de realizarlos en los pacientes. Asimismo, y en cuanto al manejo psicológico del paciente y otros aspectos de indole moral y ético relacionados con el abordaje del mismo, me gustaría introducir y llevar a cabo en un futuro CIMA juego de roles para reforzar desde otro prisma más dinámico todo lo contemplado en el mapa de contenidos.

Ciclos de Mejora en el Aula (2020). Experiencias de Innovación Docente de la US Esta obra se distribuye con la licencia Creative Commons 


\section{Aspectos de la experiencia que se pretenden incorporar a toda la práctica docente Habitual}

- Desarrollar los contenidos de cualquier asignatura tomando como hilo conductor los conocimientos de partida del alumnado.

- Estimular en el alumnado autonomía que les permita generar espíritu crítico y tomar decisiones.

- Eliminar contenido no estructural para permitir dedicar más tiempo al contenido realmente importante.

- Emplear actividad de contraste como medio metodológico imprescindible para el aprendizaje del alumnado.

\section{Principios Didácticos argumentados que han guiado la experiencia presente y que deben permanecer en el futuro}

Los principios didácticos que han guiado el presente y deben permanecer en el futuro se basan en el cambio del docente como transmisor del conocimiento en un facilitador o guía del proceso de aprendizaje del alumnado, generando escenarios similares a las prácticas clínicas de manera que el alumnado desarrolle pensamiento crítico e investigador. Dichos principios se detallan a continuación:

1. Enseñanza personalizada: la enseñanza se adapta al alumnado y no el alumnado a ella. Esto implica conocer los conocimientos de partida del alumnado para determinar los modelos mentales de los mismos que nos marcarán el desarrollo de los contenidos de enseñanza y el modelo metodológico a llevar a cabo. Todo esto se traduce, en un dinamismo continuo en el aula que será diferente no sólo en cada grupo, sino que si fuera posible en cada alumno y alumna según su modelo mental detectado (Porlán y Rivero, 2017).

Ciclos de Mejora en el Aula (2020). Experiencias de Innovación Docente de la US Esta obra se distribuye con la licencia Creative Commons 
2. A veces menos, es más: abordar una gran cantidad de contenidos sin tener en cuenta los modelos mentales del alumnado carece de sentido y lógica. Impartir muchos contenidos no implican que el alumnado lo aprenda. Por ello, los contenidos se deben diseñar en función de las necesidades del alumnado, fomentando la estructuración de los mismos de forma clara y ordenada a través de su presentación bajo mapas, problemas o preguntas que despierten en el alumnado interés, motivación y entrega en el proceso de aprendizaje, destacando en el diseño de los contenidos aquellos que insten al alumnado al aprendizaje constructivo y no memorístico.

3. Formulación de preguntas, actividades de contrastes y aprendizaje basado en problemas reales son medios metodológicos fundamentales e imprescindibles para el aprendizaje del alumnado. Hay que fomentar el espíritu investigador y crítico en el alumnado para que, con nuestra guía, construya de forma firme y autónoma su aprendizaje. Para ello, es fundamental darle tiempo de trabajo dentro y fuera del aula y unos objetivos claros desde el inicio del curso que les permita tomar decisiones y adquirir responsabilidades sobre el proceso de enseñanza-aprendizaje, es decir, no solo mostrarle qué se les va a enseñar y cómo sino también qué esperamos de su parte (Finkel, 2008).

4. Tener claro desde el inicio cuáles son los obstáculos que el alumnado tiene para avanzar en el proceso de aprendizaje es fundamental para el éxito en la enseñanza. Para ello, es sumamente necesario realizar el diagnóstico inicial a través de los cuestionarios iniciales y desarrollar las escaleras de aprendizaje y evaluación en las que plasmaremos también la evolución del alumnado en el aprendizaje tras el diagnóstico final (cuestionarios finales) y así, poder evaluar el diseño didáctico. Es muy importante que 
el alumnado evalúe también y partícipe en el diseño didáctico y en el desarrollo de contenidos, así como en nuestra labor como docentes, para lo que necesitamos obtener su opinión sobre los diferentes aspectos a evaluar (docente, modelo metodológico, etc) y tenerla en cuenta para la reformulación de todo lo que hubiera que cambiar para garantizar el máximo aprendizaje.

5. He descubierto que el diario del profesor, es de suma importancia y necesidad para el docente: los pensamientos hasta que no te materializan de forma escrita $u$ oral no adquieren cuerpo ni forma por lo que necesitamos anotar todo lo que observamos en el aula ya que ello nos ayudará a tener una visión detallada e integral de nuestro día a día y así, poder establecer fortalezas y debilidades de lo que vamos ejecutando para reformular constantemente nuestra labor como docentes (De Alba y Porlán, 2017).

Ciclos de Mejora en el Aula (2020). Experiencias de Innovación Docente de la US Esta obra se distribuye con la licencia Creative Commons 
Palabras clave: Clínica en Operatoria Dental y Endodoncia, Grado en Odontología, Docencia Universitaria, Experimentación Docente Universitaria, Prácticas Clínicas Pacientes.

Keywords: Dental Operative Clinic and Endodontics, Dentistry Degree, University Teaching, University Teaching Experimentation, Patient Clinical Practices.

\section{Referencias bibliográficas.}

Finkel, D. (2008). Dar clase con la boca cerrada. Valencia: Publicaciones Universitat de Valencia.

Porlán, R., de Alba, N. (2017). La metodología de enseñanza. En R. Porlán y cols. (Eds.). Enseñanza Universitaria. Cómo mejorarla. (pp 37-54). Madrid: Ediciones Morata.

Rivero, A., Porlán, R. (2017). La evaluación en la enseñanza universitaria. En R. Porlán y cols. (Eds.). Enseñanza Universitaria. Cómo mejorarla. (pp 73-92). Madrid: Ediciones Morata.

Ciclos de Mejora en el Aula (2020). Experiencias de Innovación Docente de la US Esta obra se distribuye con la licencia Creative Commons 